THE ASTROPHYSICAL JOURNAL, 334:95-103, 1988 November 1

(C) 1988. The American Astronomical Society. All rights reserved. Printed in U.S.A.

\title{
JET PRECESSION IN ACTIVE GALAXIES
}

\author{
Nico Roos \\ Sterrewacht, Leiden \\ Received 1987 May 13; accepted 1988 February 2
}

\begin{abstract}
The formation and evolution of massive binary black holes in the nuclei of galaxies is investigated in the context of the loss-cone filling model for the fueling of massive black holes in active galactic nuclei during the final stage of galaxy mergers. The presence of a secondary hole in a galactic nucleus may become manifest through precession of a jet propagating along the rotation axis of the primary, more massive hole during a period of activity (see Begelman, Blandford, and Rees). We derive a relation between the precession period of the jet and the fueling rate of the central hole. During the final, short phase of high central activity $\left(<10^{6} \mathrm{yr}\right)$ the precession period will decline rapidly and precession periods of order $10^{3} \mathrm{yr}$ will be attained. This may explain why jets in luminous quasars like 3C 273 and 3C 345 often are strongly curved on the VLBI scale while the position angle variations observed in the arcsec scale radio structure are indicative of much longer precession periods. Investigation of the observational data for a number of well-studied radio galaxies and quasars indicates that the characteristic time for position angle variation may indeed be correlated with the level of central activity in the sense predicted by the model. The observations further suggest that rapidly precessing jets propagate within narrow precession cones, possibly because these jets become collimated along the precession cone axis. It is pointed out that rapid precession in powerful quasars might have important implications for unified schemes based on the relativistic beaming hypothesis.
\end{abstract}

Subject headings: galaxies: interactions — galaxies: jets - galaxies: nuclei — radio sources: extended

\section{INTRODUCTION}

The existence of massive binary black holes in the nuclei of active galaxies was first proposed by Begelman, Blandford, and Rees (1980, hereafter BBR). The spin axis of each binary component will undergo geodetic precession about the toal angular momentum of the binary system. Collimated beams of plasma ejected along the spin axis of a central massive hole are thought to fuel the extended radio structures straddling the nucleus of most radio galaxies. Precession of the hole's spin axis may then account for the inversion symmetry often observed in these radio sources (e.g., Miley.1980). BBR discuss the formation of a central massive binary hole as a result of a merger of two galaxies both containing a central massive hole. During the merger the two holes will lose orbital energy through stellar dynamical processes until a loss cone is formed in the stellar distribution around the binary. This impedes further evolution, and the binary will settle in the nucleus of the new galactic system at a separation of the order of parsecs. The corresponding precession periods for black holes of $10^{7}-10^{8} M_{\odot}$ are of the right order to explain the inversion symmetry in extended radio sources, and also the complementary emission patterns that have been ascribed to alternating side ejection (Roos and Meurs 1987).

Jet precession might also account for the bending and misalignment of milli-arcsec scale jets observed in the compact cores of some powerful quasars like 3C 273 and 3C 345 (Linfield 1981). A number of observational problems with this interpretation of the milli-arcsec curvature will be discussed in $\S \mathrm{V}$. Here we will mention two problems that are of particular interest to the binary black hole model. The arcsec scale jet in 3C 273 is almost straight. If we attribute the small amplitude wiggle recently found by Davis $e t$ al. (1985) to precession, its period of $\sim 3 \times 10^{4} \mathrm{yr}$ would imply a binary separation of order $10^{-1} \mathrm{pc}$. The milli-arcsec curvature requires a precession period of $\sim 500 \mathrm{yr}$ (BBR) yielding a binary separation which is almost one order of magnitude smaller. The situation in $3 \mathrm{C} 345$ is similar. The following problems now arise:

1. Which process causes the binary orbit to shrink to radii smaller than $1 \mathrm{pc}$, or how does the binary cross the gap from the separation where stellar dynamical processes become unimportant to separations of $\sim 10^{-2} \mathrm{pc}$ where the binary may lose energy and merge due to the emission of gravitational waves. BBR suggest that infall of gas onto the binary might lead to orbital evolution in this regime. The gas may be flung out of the binary system or be accreted by the larger hole. In either case the binary orbit shrinks on a time scale given by the ratio of the central black hole mass and the mass inflow rate. Rapid binary evolution on a time scale of order of $10^{6} \mathrm{yr}$ as may have occurred in 3C 273 would thus require large mass inflow rates of $10^{2} M_{\odot} \mathrm{yr}^{-1}$ or more.

2. Short precession times of $10^{3} \mathrm{yr}$ imply binary lifetimes of order $10^{6} \mathrm{yr}$ for reasonable black hole masses. The probability to observe a massive binary with such a short lifetime seems unacceptably low unless the formation of the binary black hole is somehow related to the quasar phenomenon.

These considerations suggest that during the most active phase of an active galactic nucleus (AGN) there is an inflow of $\sim 10^{2} M_{\odot}$ $\mathrm{yr}^{-1}$ onto the binary orbit which may account for both the rapid binary evolution and the fueling of the central black hole, as some fraction of the inflowing mass is consumed by one or both black holes. The high mass inflow rate of $10^{2} M_{\odot} \mathrm{yr}^{-1}$ implies that the mean density of the inflowing matter is an appreciable fraction of the galactic density in the nucleus. This seems difficult to achieve in models where the inflowing mass is in the form of gas originating from outside the galactic nucleus. Filling of the loss cone in the 
stellar distribution around the binary during a galaxy merger seems a better alternative since it makes full use of the high stellar mass density of the galactic nucleus.

In previous papers we have investigated the fueling of single massive black holes in the nuclei of galaxies during galaxy mergers and estimated the local luminosity function of active galaxies as well as its cosmological evolution (Roos $1981 b, 1985 a, b$, hereafter Papers I, II, and III). Most galaxies on the bright side of the galaxy luminosity function may have undergone several mergers with smaller galaxies since their formation. Elliptical galaxies as well as the spheroidal components of disk galaxies may have formed as a result of this process (Toomre and Toomre 1972; Roos 1981a). If the binary holes that are expected to form as a result of mergers do not evolve significantly by other than stellar dynamical processes should perhaps assume that galaxies contain massive binaries instead of single black holes. Systems of three or more black holes are not stable and will quickly evolve toward a binary system via ejection of the less massive holes. The assumption of binary black holes in galactic nuclei allows a more self-consistent model for the fuelling of central black holes during galaxy mergers, because, as we will show, a merger will lead to further evolution and finally coalescence of a preexisting central binary and the production of a new stable binary at a similar separation as the initial binary.

In $\S$ II we first estimate the final separation of massive binaries that are formed as a result of galaxy mergers. In $\S$ III we describe the evolution of such a binary during a subsequent merger event. We will see that in the early stages of activity the precession period of the central black hole's axis will remain constant and much larger than the period of activity. When the fueling rate reaches a particular level the binary starts evolving, and during the final short period of high activity the precession period decreases rapidly. In $\S$ IV we compare our predictions regarding the correlation between precession period and fueling rate with observational data. Some obseevational problems concerning rapid precession in radio sources will be discussed in $\S$ V. In $\S$ VI we summarize and discuss our main results.

\section{MASSIVE BINARIES IN GALACTIC NUCLEI}

The various stages in the formation of a central binary black hole from two massive black holes in the nuclei of a pair of merging galaxies have been discussed by BBR. They show that stellar dynamical processes operate to remove orbital energy from the binary until a separation of the order of parsecs is reached. The orbital radius of the binary will be determined by the condition that the orbital energy which can be transferred via the slingshot mechanism to stars passing through its orbit is comparable to the binding energy of the binary. The binary will then create a loss cone in the stellar distribution of the galaxy. Further dynamical evolution of the binary is controlled by the very slow rate at which stars can diffuse into loss cone orbits via two-body relaxation.

The radius of the binary orbit resulting from a merger is quite insensitive to the form of the stellar density distribution in the galactic nucleus or to the mass of the secondary black hole. Within the cusp radius $r_{h}$, defined by $r_{h}=G M / \sigma^{2}$, where $G$ is the gravitational constant and $\sigma$ is the one-dimensional velocity dispersion of stars in the nucleus of the galaxy, the density and velocity
dispersion vary with $r^{-7 / 4}$ and $r^{-1 / 2}$ respectively dispersion vary with $r^{-7 / 4}$ and $r^{-1 / 2}$, respectively (Bahcall and Wolf, 1976). We will assume that the density distribution outside the cusp varies approximately with $r^{-2}$ as in the Milky Way (Oort 1977). For an isotropic velocity distribution the total mass of stars that move on orbits passing through the binary orbit $r_{b}$ is given by

$$
M_{\text {cone }}\left(r_{b}\right) \approx 6 M_{\text {cusp }} r_{b} / r_{h}
$$

where $M_{\text {cusp }}$ is the stellar mass within $r_{h}$ which is about equal to $M+m$. The fraction of the orbital energy that the binary transfers to stars of mass $m_{*}$ is estimated to be $\sim 2 m_{*} /(M+m)$ from numerical experiments (Paper I). This result, which differs from the expression used by BBR, has been confirmed by the extensive numerical three-body studies of Hills (1983). The binary evolution will stop when $2 M_{\text {cone }}\left(r_{b}\right) /(M+m) \approx 1$, or

$$
r_{b} / r_{h} \approx 0.1(M+m) / M_{\text {cusp }} \approx 0.1 .
$$

Mergers among bright galaxies probably occurred frequently enough to produce massive binaries in the nuclei of most galaxies populating the bright side of the galaxy luminosity function. Infall of gas onto a binary during periods of activity could lead to further evolution and finally coalescence of binaries. However, if we take the view that such periods of activity are also caused by galaxy mergers, then we should assume that the nuclei of most bright galaxies contain massive double black holes with separations of the order of one-tenth of the cusp radius. Note that a merger of two galaxies both containing a double black hole will also result in the formation of a stable binary because the less massive holes will quickly be expelled from the four-body system that is formed
during the merger.

At a binary separation $r_{b}$ the precession time for the more massive hole is given by ${ }^{1}$

$$
t_{\text {prec }}=9.3 \times 10^{8} r_{b}^{5 / 5}(M / m) M^{-3 / 2} \mathrm{yr}
$$

With equation (2) and $r_{h}=10 M / \sigma^{2}$ this yields for the wide binaries in galactic nuclei produced by mergers:

$$
t_{\text {prec }}=9.3 \times 10^{8}(M / m) M \sigma^{-5} \mathrm{yr},
$$
which may indeed provide the explanation for the long precession times inferred from inversion symmetric extended radio sources
as pointed out by BBR (see also $§ I V$ ).

\section{BINARY EVOLUTION AND FUELING DURING GALAXY MERGERS}

We now assume that the nuclei of bright galaxies contain massive binary black holes and study the simultaneous evolution of such a binary and the fueling of the central hole during a (subsequent) galaxy merger.

${ }^{1}$ If not specified otherwise, distances, masses, and velocities will be expressed in parsecs, $10^{8} M_{\odot}$ and $200 \mathrm{~km} \mathrm{~s}^{-1}$, respectively. 
As the intruding galaxy spirals inward under the influence of dynamical friction, an increasing number of stars will be scattered into the loss-cone in the stellar distribution around the central massive binary. A fraction of $\sim r_{T} / r_{b}$ of these stars will pass at a separation smaller than $r_{T}$ of the more massive hole where they will be tidally disrupted and consumed by the hole. The fate of stars tidally disrupted near a massive black hole has recently been discussed by Rees (1988). For solar-type stars the distance $r_{T}$ is given by

$$
r_{T}=2 \times 10^{-5} M^{1 / 3}
$$

Hills (1975) noted that for black hole masses larger than $\sim 10^{9} M_{\odot} r_{T}$ becomes smaller than the Schwarzschild radius of the hole and stars may be swallowed whole. Initially the number of stars that interact with the binary will be quite small, and the binary evolves on a time scale $t_{\mathrm{ev}}^{\text {bin }}$ defined by $\left(r_{b}^{-1} d r_{b} / d t\right)^{-1}$, which is much larger than the time scale $t_{\mathrm{ev}}^{\text {sat }}$ for the evolution of the satellite's orbit due to dynamical friction. As the intruding galaxy approaches the cusp radius the two dynamical times become equal. Thereafter the binary evolution is driven by the evolution of the satellite, and $r_{b}$ is determined by the separation of the two galaxies through $t_{\mathrm{ev}}^{\text {bin }}=t_{\mathrm{ev}}^{\text {sat }}$, until the loss of orbital energy via the emission of gravitational waves occurs faster than via interactions with stars.

We will now make these considerations more quantitative. Our treatment of the loss-cone filling process will be similar to that given in Paper I, to which we refer for details. We start by estimating the so called "critical radius" for the scattering of stars into loss-cone orbits by the intruding galaxy. This is the smallest distance from the center of the (more massive) galaxy where the tidal force exerted by the intruder on the stellar orbits in the central regions of the galaxy is just strong enough to fill the loss cone of the central binary orbit in one orbital period of the stars at that distance. At radii larger than $r_{\text {crit }}$ there is no longer a loss-cone, and the velocity distribution of the stars may be considered as isotropic. Following the approach of Paper I we find, using the impulsive approximation for the deflection of stellar orbits by the intruder which is moving at a distance $r$ from the center of the more massive galaxy:

$$
r_{\text {crit }}=0.7\left\{\begin{array}{ll}
\left(M / M_{\text {sat }}\right)^{1 / 3} r_{b}^{1 / 6} r_{h}^{-1 / 6} r & \text { for } r_{\text {crit }}>r_{h} \\
\left(M / M_{\text {sat }}\right)^{2 / 7} r_{b}^{1 / 7} r^{6 / 7} & \text { for } r_{\text {crit }}<r_{h}
\end{array} .\right.
$$

For an isotropic velocity distribution the flux of stars at a distance $d$ from the galactic center passing through the binary orbit is given by

$$
F(d) / M_{0} \mathrm{yr}^{-1}=2 \times 10^{4} M \sigma r_{b}\left\{\begin{array}{ll}
d^{-2} & d>r_{h} \\
d^{-5 / 4} r_{h}^{-3 / 4} & d<r_{h}
\end{array} .\right.
$$

The actual inflow rate of stars through the binary orbit is found by setting $d$ equal to $r_{\text {crit }}$ in equation (7). The binary evolution time due to three-body interactions with stars brought into loss-cone orbits by an intruding galaxy is now

$$
t_{\mathrm{ev}}^{\mathrm{bin}}=10^{8} \mathrm{yr} F^{-1}\left(r_{\mathrm{crit}}\right)(M+m) / 2 .
$$

The dynamical evolution time of the satellite assumed to be moving on a circular orbit is approximately given by

$$
t_{\mathrm{ev}}^{\mathrm{sat}}=10^{4} \mathrm{yr}\left\{\begin{array}{ll}
r \sigma^{-1} M_{g}(r) / M_{\mathrm{sat}}(r)=10^{4} r \sigma^{-1} f^{-1} & \text { for } r>r_{h} \\
\sigma^{-1}\left(r / r_{h}\right)^{-1} r_{h} & \text { for } r<r_{h}
\end{array} .\right.
$$

As in Paper I we have assumed here that the two merging galaxies have similar density profiles which implies that the mass ratio outside $r_{h}$ is given by $f=M_{\text {sat }}\left(r_{h}\right) / M$. Inside the cusp radius the dynamical evolution is independent of the mass ratio (see eq. [2]). The binary orbit does not evolve appreciably until $t_{\mathrm{ev}}^{\text {bin }} \approx t_{\mathrm{ev}}^{\text {sat }}$. Thereafter the binary evolution time remains equal to the satellite's evolution time and the orbital radius is given by

$$
r_{b}=0.03 \begin{cases}f^{1 / 2} r_{h}^{1 / 2} r^{1 / 2} & \text { for } r>r_{h} \\ f^{-10 / 23} r_{h}^{-25 / 23} r^{48 / 23} & \text { for } r<r_{h}\end{cases}
$$

until energy losses due to the emission of gravitational waves become more important.

In Figure 1 the relations between the dynamical evolution times and the orbital radii of the massive binary and the satellite galaxy are illustrated for a galaxy having a one-dimensional velocity dispersion of $200 \mathrm{~km} \mathrm{~s}^{-1}$ and a central binary black hole with masses of $10^{8} M_{\odot}$ and $10^{7} M_{\odot}$. We conclude that preexisting central binary black holes will undergo further dynamical evolution and finally coalesce as a result of a galactic merger event while at the same time a new long-lived binary with an orbital radius given by equation (2) may be formed.

Perhaps the most interesting result of the model is that it yields a relation between the precession period of a central black hole and the level of activity set by the consumption of stars tidally disrupted near the central hole. The probability that a star passing once through the binary orbit will be captured by the more massive hole will be of order $r_{T} / r_{b}$. However, some fraction of these stars will make several orbital revolutions around the center of mass of the binary before being ejected. J. G. Hills estimates from his numerical simulations that a considerable fraction of the stars passing through the binary orbit, especially those passing close to the more massive hole, will be captured in long-lived orbits. This would increase the chance that they become tidally disrupted before being ejected, by some factor $D$ which is larger than 1 but probably not larger than 10 . The tidal disruption rate which we assume equal to the fueling rate of the more massive hole is then given by

$$
F_{M}=D F\left(r_{\text {crit }}\right) r_{T} / r_{b}
$$




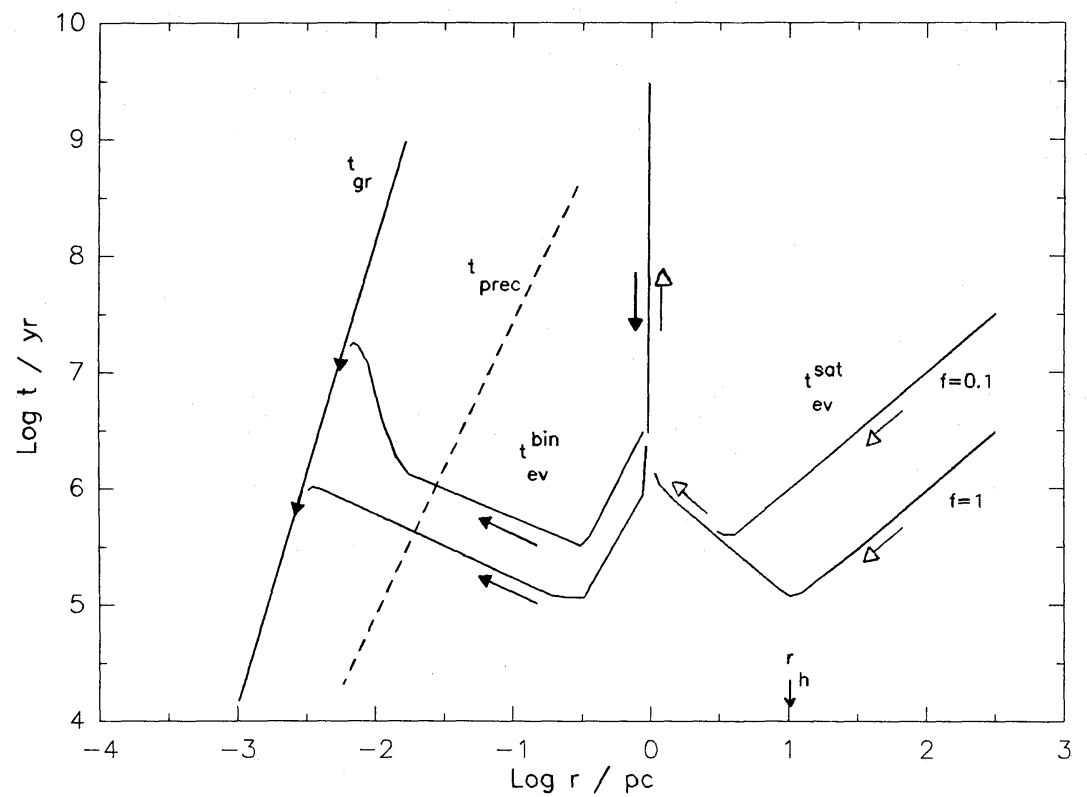

Fig. 1.-Evolution times as a function of orbital radius for the central binary black hole $\left(r=r_{b} ;\right.$ filled arrows) and for the intruding galaxy $\left(r=r_{\text {sat }} ;\right.$ open arrows) during a galaxy merger. The masses of the central binary are $10^{8}$ and $10^{7} M_{\odot}$, and the central one-dimensional velocity dispersion of the galaxy is $200 \mathrm{~km} \mathrm{~s} \mathrm{~s}^{-1}$. Dashed line gives the evolution of the precession time of the more massive hole.

Note that this is almost identical to the expression derived in Paper I for the fueling of a single hole during a merger. The definition of $r_{\text {crit }}$ differs from that in Paper I because $r_{T}$ is here replaced by $r_{b}$. As a result, $r_{\text {crit }}$ is larger by about one order of magnitude and is almost equal to $r$. Combining equations (5), (6), (10), and (11) yields

$$
F_{M} / M_{\odot} \mathrm{yr}^{-1}=\left\{\begin{array}{ll}
1.7 \times 10^{-4} D M^{4 / 3} \sigma f^{2} r_{h} r_{b}^{-3} & r>r_{h} \\
1.0 D M^{4 / 3} \sigma f^{5 / 24} r_{h}^{-71 / 48} r_{b}^{-25 / 48} & r<r_{h}
\end{array} .\right.
$$

Stars from the perturbing galaxy will also contribute to the inflow rate of stars onto the binary. This contribution will generally be smaller than equation (12) unless the perturber has a higher central star density than the perturbed system.

The binary does not evolve until the intruding galaxy is at a separation of $10^{2} M \sigma^{-2} f^{-1} \mathrm{pc}$. The precession period is then given by equation (4) and the tidal disruption rate is $1.4 \times 10^{-3} M^{-2 / 3} \sigma^{5} f^{2} M_{\odot} \mathrm{yr}^{-1}$. Thereafter the precession period and tidal disruption rate are related through

$$
t_{\mathrm{prec}} \mathrm{yr}^{-1}=\left\{\begin{array}{ll}
5 \times 10^{6} M^{4 / 9}(M / m) f^{5 / 3} \sigma^{-5 / 6}\left(F_{M} / D\right)^{-5 / 6} & F_{M}<F_{M}^{*} \\
34 M^{-11 / 5}(M / m) f \sigma^{15}\left(F_{M} / D\right)^{-24 / 5} & F_{M}>F_{M}^{*}
\end{array} .\right.
$$

The change of slope occurs at $r=r_{h}$ yielding

$$
\begin{aligned}
r_{b}^{*} & =0.03 f^{1 / 2} r_{h}=0.3 f^{1 / 2} M \sigma^{-2}, \\
t_{\mathrm{prec}}^{*} & =6 \times 10^{7} f^{5 / 4}(M / m) M \sigma^{-5} \mathrm{yr}, \\
F_{M}^{*} & =0.05 D M^{-2 / 3} f^{1 / 2} \sigma^{5} M_{\odot} \mathrm{yr}^{-1} .
\end{aligned}
$$

Apart from the numerical factor $F_{M}^{*}$ is the same as the fueling rate for $r_{\text {crit }}=r_{h}$ in Paper I, which is not surprising because here we have $r \approx r_{\text {crit }}$. In Paper II we identified it with the break in the local bivariate radio luminosity functions of galaxies from which we found $^{2}$

$$
\log P_{1.4 \mathrm{GHz}}^{*}=26.8+\log F_{M}^{*}
$$

and $M \propto \sigma^{15 / 2}$.

In Figure 2 we have drawn some evolution tracks in the precession period versus tidal disruption rate diagram for some typical black hole masses and galaxy mass ratios. We have also sketched the relation between the tidal disruption rate and the time scale given by equation (9) during which the AGN is active at that fueling level. This time scale multiplied by the jet velocity determines the scale of the extended radio structures that are formed by the central engine at the corresponding precession period and fueling level. The ratio of the two time scales determines the number of precession periods that might be discernable in radio structures on that scale. Some interesting conclusions can be drawn from this figure.

1. Below $F_{M} \approx 10^{-1.5} D M_{\odot} \mathrm{yr}^{-1}$ most sources will have $t_{\mathrm{prec}}>t_{\mathrm{ev}}^{\mathrm{sat}}$. Precession periods may range from $10^{6}$ to $10^{9} \mathrm{yr}$.

\footnotetext{
${ }^{2}$ In Paper II this equation contains a typing error.
} 


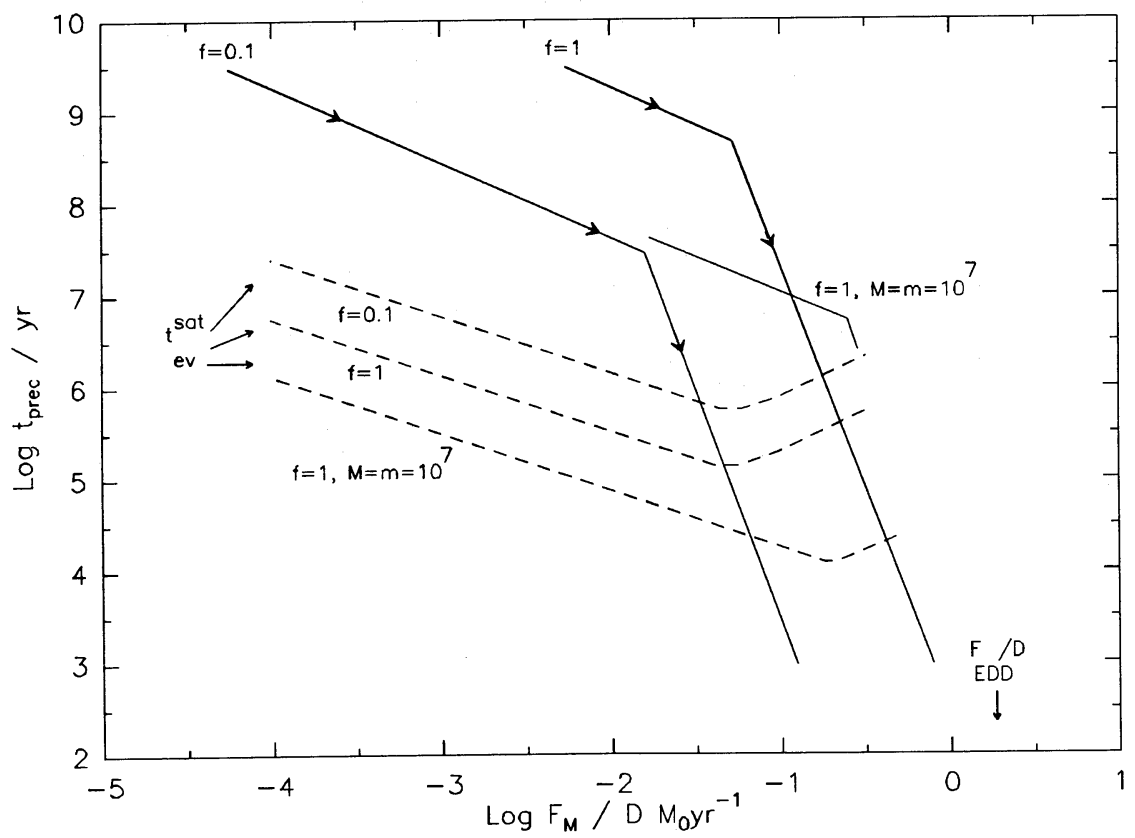

FIG. 2.-Precession period vs. fueling rate (assumed equal to tidal disruption rate) for a merger of a galaxy with one-dimensional velocity dispersion of $200 \mathrm{~km}$ $\mathrm{s}^{-1}$ with a smaller galaxy. The black hole masses are $M=10^{8} M_{\odot}$ and $m=10^{7} M_{\odot}$ unless specified otherwise. The evolution time scale for the orbit of the satellite galaxy is given by the dashed lines, $F_{\text {EDD }}$ is the Eddington limit for a mass to energy conversion of $10 \%$, and $D$ is defined in $\S$ III.

2. AGNs with $F_{M}>10^{-1.5} D M_{\odot} \mathrm{yr}^{-1}$ may exhibit strong jet curvature on scales smaller than $\sim 10^{5} v_{\text {jet }} / c$ light year, while there may be little curvature on larger scales. These sources are in their final stage of activity during which the precession period rapidly declines, and precession periods of $10^{3}$ yr may be attained.

This rapid decline of $t_{\text {prec }}$ during the final most luminous phase of an AGN, lasting for $\sim\left(10^{4}-10^{6}\right) M \sigma^{-3} \mathrm{yr}$, might explain why the precession period in $3 \mathrm{C} 273$ appears to have decreased by a few orders of magnitude during the last million years.

\section{DO POWERFUL AGNS PRECESS FASTER?}

In this section we will investigate the observational evidence for an inverse correlation between precession period and fueling rate of the central engine in AGNs. The observational quantities we assume to be correlated with the fueling rate of the central black hole in an AGN are the optical (nonstellar) luminosity and the radio brightness of the extended, steep spectrum components of the radio source associated with the AGN. The optical luminosity may be related more directly to the fueling rate than the radio luminosity because a strong radio source may derive its energy from the rotational energy of a spinning hole at low fueling levels (e.g., Begelman, Blandford, and Rees 1984). The radio emission of the central cores of radio sources is likely to be affected by relativistic beaming (Blandford and Königl 1979; Orr and Browne 1982). The one-sidedness and superluminal motions of milliarcsec jets, as well as variability arguments and the lack of inverse Compton X-ray emission, all speak in favor of relativistic motion on the parsec scale. On larger scales the propagation velocities of radio components are probably lower. The resolved steep spectrum components of extended radio sources is likely to move not faster than $\sim 0.2 c$ with respect to the intergalactic medium (Longair and Ryle 1979). Also, jets in weak radio sources $\left(P_{1.4 \mathrm{GHz}}<10^{25} \mathrm{WHz}^{-1}\right)$ are mainly two-sided, indicating that relativistic effects are not very important. Among these sources we often find head-tail radio galaxies whose jets are probably bent due by ram pressure as the galaxy moves through the intergalactic medium in a cluster of galaxies. Jets in luminous radio sources $\left(P_{1.4} \mathrm{GHz}>\right.$ $10^{25} \mathrm{WHz}^{-1}$ ) are mainly one-sided. Many of these jets may well be moving in our direction with a velocity close to the speed of light (Bridle and Perley 1984). We should therefore keep in mind that the radio brightness of the extended components of some (coredominated) sources may to some extent be Doppler boosted.

The time scale on which the ejection axis in a radio source changes direction is not easily estimated either. The main uncertainties are the jet's velocity and orientation with respect to the observer. Perhaps the best way of estimating the precession period of a radio source is by fitting its radio map with a precessing jet model (Icke 1981; Gower et al. 1982). Several sources have satisfactorily been modeled in this way (3C 129, Icke 1981; 2300-189, Hunstead et al. 1983; 4C 29.47, Condon and Mitchell 1984; NGC 6251, Jones et al. 1986; 3C 418, Muxlow, Jullian, and Linfield 1983; further sources in Fig. 3: Gower et al. 1982). The resulting precession periods are shown by open symbols in Figure 3. The four most powerful sources in this group are all flat spectrum core-dominated sources. We must be careful not to overestimate the radio power of the extended components of these sources, especially because they appear to have short precession periods. From the distribution of $R$ (core flux density/flux density of extended emission) as obtained by Browne and Perley (1986; see their Fig. 4) we see that most core-dominated sources have $R<10$. We feel that the radio power of the extended components in these sources is biased against high radio power if we choose $R=10$, which is the value adopted for these sources in Figure 3. Note that the maximum value of $R$ is $\sim 50$. Even if all four sources would have such a high value of $R$ it would not invalidate the conclusions we will draw at the end of this section.

The sources discussed so far were selected for their precession-like appearance. Next we will estimate precession periods for the 


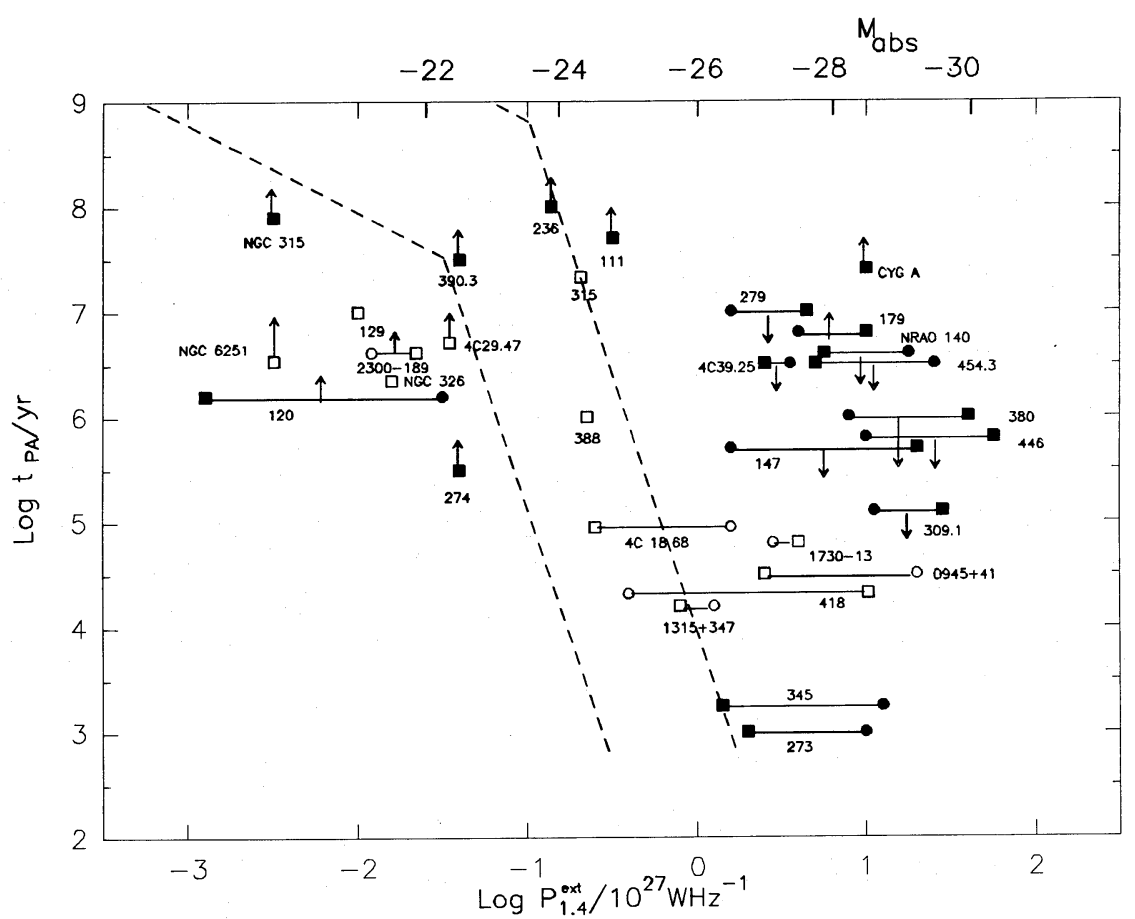

FIG. 3.-Estimated precession periods in radio sources vs. radio power at $1.4 \mathrm{GHz}$ of the extended radio components (see $\S$ IV). The radio powers are shown as squares. The optical luminosities of the Seyferts and QSOs (Veron-Cetty and Veron 1985) are indicated by dots (upper scale). The precession periods obtained by fitting precessing jet models to radio maps are indicated by open symbols. Filled symbols are precession periods for the sources in the lists of Readhead et al. (1978) and Browne et al. (1982). 3C numbers, positions and other source names are indicated. Dashed lines are the same as the full lines in Fig. 2 for a constant ratio of fueling rate over radio power as given by eq. (17).

samples of extragalactic radio sources studied by Readhead et al. (1978) and by Browne et al. (1982). We have chosen these sources because all have all been observed using Very Long Baseline Interferometry (VLBI) and position angles with respect to the central source have been determined for radio components on the milli-arcsec scale as well as on the arcsec scale. The radio power of the extended components were obtained from Browne et al. (1982), Readhead et al. (1978), and Schilizzi and de Bruyn (1983). Suppose we observe a change in position angle $\Delta \mathrm{PA}_{\mathrm{obs}}$ over the projected angular size $\theta$ of a radio source. We can express the characteristic time scale $t_{\triangle \mathrm{PA}}$ for the change in position angle in this source as a fraction $f_{\mathbf{P A}}$ of the jet travel time:

$$
t_{\Delta \mathrm{PA}} \approx f_{\mathrm{PA}} \theta d_{A} / v_{\mathrm{app}}
$$

$v_{\text {app }}$, the apparent velocity of the jet or outmoving radio blobs with respect to the central engine, is given by

$$
v_{\text {app }}=v_{\text {jet }}(1-\beta \cos i)^{-1} \sin i,
$$

where $\beta=v_{\text {jet }} / c$ and $i$ is the angle between the jet direction and the observer's line of sight. The angular size distance ${ }^{3}$ (Weinberg 1972 ) is denoted by $d_{A}$.

In the case of precession, $f_{\mathrm{PA}}$ will be the fraction of the precession cycle (or the number of precession cycles) discernible in the radio map. In order to estimate $v_{\text {app }}$ and $f_{\mathrm{PA}}$ we need to make assumptions about $v_{\text {jet }}, i$, and in the case of precession also for $\psi$, the opening half angle of the precession cone. Following Orr and Browne (1982) we assume that core-dominated sources have a small inclination to the line of sight and jet velocity close to the speed of light. The remaining freedom of choice for $f_{\mathrm{PA}}, i$, and $v_{\text {jet }}$ is used to obtain lower limits to $t_{\triangle \mathrm{PA}}$ at low radio power and upper limits at high radio power in order to minimize a possible inverse relation between $t_{\triangle \mathrm{PA}}$ and central activity of the kind predicted in the previous section. This was done as follows.

1 . For the sources that are not core-dominated (symmetric sources in the terminology of Readhead et al. 1988) we use $f_{\mathrm{PA}}=$ $\pi / \Delta \mathrm{PA}_{\mathrm{obs}}, i=90^{\circ}$, and $v_{\text {jet }}=c ; \Delta \mathrm{PA}_{\mathrm{obs}}$ was estimated from the difference in position angles on the arcsec scale and the VLBI scale. This yields a lower limit to $t_{\mathrm{PA}}$, because: (1) for several sources we have only an upper limit for $\Delta \mathrm{PA},(2)$ the majority of these sources has low radio power and $v_{\text {jet }}$ may well be smaller than $c$, and (3) sin $i$ will be smaller than 1 . One could raise the objection that the position angle in some of these sources might vary within very narrow limits on a time scale which is shorter than our lower limit. Recently Jones et al. (1986) have shown that the wiggles in the arcsec scale structure of, for instance, NGC 6251 can be modeled by a precessing jet with $t_{\text {prec }}=1.8 \times 10^{6} \mathrm{yr}$ and $\psi=8^{\circ}$. An even smaller wiggle was found in the large-scale jet of $3 \mathrm{C} 273$ by Davis $e t$ al. (1985). In $\S \mathrm{V}$ we will digress on the possibility of small $\psi$ precession. For the moment we will discard the possibliity of periodic position angle variations over a range smaller than $\sim 5^{\circ}$.

2. The core component of core-dominated sources is probably oriented close to the line of sight: observation of superluminal motion in many of these sources indicates that $i \approx \gamma^{-1}$, with a Lorentz factor $\gamma=\left(1-\beta^{2}\right)^{-1 / 2}$ of $\sim 5$ (Cohen and Unwin 1984). The

${ }^{3}$ We use $H_{0}=50 \mathrm{~km} \mathrm{~s}^{-1} \mathrm{Mpc}^{-1}$ and $q_{0}=\frac{1}{2}$. 
mean $\triangle \mathbf{P A}_{\mathrm{obs}}$ in these sources is $\sim 75^{\circ}$, which would correspond to a deprojected change in the position angle of $\sim 20^{\circ}$. Schilizzi and de Bruyn (1983) concluded from the observed size of the extended structures around the dominant cores of several superluminal sources that the change in deprojected position angle is probably even larger than $30^{\circ}$. For these sources we have chosen $f_{\mathrm{PA}}=\pi / 0.35, i=20^{\circ}$, and $v_{\text {jet }}=c / 3$ for the arcsec scale structure. The precession periods obtained in this way should be regarded as upper limits, because (1) the large $\triangle P A$ and the strong curvature on the VLBI scale in some of these sources suggest that several precession periods between the milli-arcsec and the arcsec scale could have been missed, (2) some sources in this group (for instance $3 \mathrm{C} 446$ and $3 \mathrm{C} 309.1)$ are one-sided. If the arcsec scale jet in these sources also has $\gamma=5$ then $v_{\text {app }}=3 c$ for $i=20^{\circ}$. The only sources we left out were 3C 371 and 3C 84 because the situation in these sources is not clear. Readhead et al. (1978) give a considerable change in position angle between the large-scale structure and the VLBI jet in 3C 371 but van der Laan et al. (1984) found that the VLBI jet is aligned with an extended structure on a still larger scale. In 3C 84 the milli-arcsec scale structure is aligned with the arcsec component, but the VLBI component closest to the core has a different position angle (Readhead et al. 1983).

3. Following BBR we have estimated $t_{\triangle \mathrm{PA}}$ in the core-dominated sources $3 \mathrm{C} 273$ and $3 \mathrm{C} 345$ from the curvature observed on the VLBI scale using $f_{\mathrm{PA}}=\gamma \pi$ and $v_{\text {app }}=\gamma c$.

The results shown in Figure 3 indicate that rapid change of position angle on a time scale smaller than $10^{5} \mathrm{yr}$ occurs only in the most powerful sources $\left(P_{1.4 \mathrm{GHz}}^{\text {ext }}>10^{26} \mathrm{WHz}^{-1}, M_{B}<-26\right)$. The fact that these sources also have higher optical luminosities than the weaker radio sources indicates that the high-energy output is due to a high fueling rate of the central hole rather than extraction of rotational energy from the hole (Begelman et al. 1984). Comparing the data points in Figure 3 with the evolution curves given by equations (13) and (14) we would expect the break in the evolution curves to occur at $P_{1.4}^{\text {ext }} \mathrm{GHz} \approx 10^{26}$ to $10^{27} \mathrm{WHz}^{-1}$, which is a factor of 3-30 higher than expected if we use equation (15) for the relation between the fueling rate and the radio power. We have drawn the evolution curves displayed in Figure 2 also in Figure 3 using

$$
\log P_{1.4 \mathrm{GHz}}=27.3+\log F_{M} / D \text {. }
$$

The model exhibits a strong decline in $t_{\text {prec }}$ at high luminosities. This makes it difficult to account for the sources like Cygnus A which combine very high radio luminosity with remarkably little variation in position angle. Note, however, that the nucleus of Cygnus A has a very low optical luminosity for its radio power, which suggests that the energy in this source is drawn from the rotational energy of the central hole while the fueling level is lower than for the optically bright quasars in Figure 3.

The model suggests that the weaker sources with precession periods near $10^{6} \mathrm{yr}$ are in smaller galaxies harboring less massive black holes of $\sim 10^{6} M_{\odot}$. This may be correct for 3C 120 and $2300-189$, but probably not for the giant ellipticals NGC 6251, NGC 326 , and $3 \mathrm{C} 274$.

Several problems remain which keep us from drawing definite conclusions from Figure 3. (1) Our sample is certainly not free of selection effects. Most of the powerful quasar sources, for instance, have strong cores, and their redshifts range from 0.5 to 1 compared to 0.01 to 0.1 for the weaker radio galaxies. Other problems are (2) small number statistics especially for the sources that appear to have a rapidly changing position angle on the milli-arcsec scale, and (3) observations of fine structure in radio sources on the left side of the diagram may reveal rapid position angle variations within a narrow range.

So far, our results indicate that rapid change of the position angle in radio sources occurs only in powerful quasars. This would not be expected if the observed curvature were due to bending of the jet by pressure gradients. If on the other hand the curvature is caused by precession of the central hole's axis, this behavior would be consistent with the predictions of the merger model discussed in the previous section.

\section{RAPID PRECESSORS: PROBLEMS AND POSSIBILITIES}

For a random orientation of the orbital angular momentum of the binary with respect to the spin axis of the most massive black hole the median value of the half angle of the precession cone is $60^{\circ}$. The large- and small-scale jets will generally have a quite different orientation with respect to the observer when the jet travel time between the two scales is comparable to or larger than a precession period. Several observations are difficult to explain if some powerful quasars would indeed be rapidly precessing:

1. The milli-arcsec jet in $3 \mathrm{C} 272$ and $3 \mathrm{C} 345$ tends to line up with the arcsec scale jet as it moves outward.

2. Subsequent observations of the VLBI component C4 moving near the core of 3C 345 indicate that it follows a curved path. Components further out appear to move on rectilinear orbits (Biretta, Cohen, and Moore 1985).

3. Core-less jets have not been found, and one-sided jets have similar radio brightnesses as the cores they are pointing at, suggesting that the Lorentz factor and orientation of the jet are not very different from the core.

4 . The distribution of opening half angles derived from model fits of relativistic precessing jets to observed brightness distributions (see $\S$ III) is strongly peaked toward small angles; nearly all sources have $\psi<20^{\circ}$. In some sources the observations seem to call for an opening angle which increases with decreasing distance from the core (Gower et al. 1982; Muxlow et al. 1983).

Small opening angles may be expected when the central hole is embedded in a rotating disk galaxy with its spin axis aligned with the rotation axis of the galaxy. A merger with a smaller galaxy containing a black hole will then produce a binary with an orbital angular momentum closely aligned with the spin axis of the more massive hole because the satellite's motion perpendicular to the disk is damped faster than its motion in the disk (Quinn and Goodman 1987).

A perhaps more attractive, but ill-understood solution, already mentioned by BBR, is to assume that the material ejected along the rapidly precessing spin axis of the central hole is collimated along the precession cone axis. We would then observe the following radio structures in a source that has become a rapid precessor during the last $10^{6} \mathrm{yr}$.

1. An extended large-scale structure formed by a slowly precessing jet over a period of $10^{6}-10^{8} \mathrm{yr}$.

2. A smaller jet-like structure directed along the precession cone axis over a distance of $10^{6} c / v_{\text {jet }}$ light year or less. The transition between this structure and the radio structure on larger scales may be quite abrupt. 
3. On the scale where the jet travel time is comparable to the short precession period $\left(10^{3}-10^{6}\right.$ yr) we expect to see curvature of the jet due to rapid precession, perhaps accompanied by bending toward the precession cone axis, as the jet moves outward.

This version of the precessing jet model for rapid precessors appears not to suffer from the problems mentioned above. Moreover, it has some interesting observational consequences. The observer's line of sight will lie within the precession cone of half of all rapid precessors if the angular momentum of the massive binary is randomly oriented with respect to the spin axis of the more massive hole. In the region where collimation along the precession axis occurs, part of the relativistic material will be propagating in a direction close to the observer's line of sight $\left(i<\gamma^{-1}\right)$. Its Dippler-boosted emission will probably outshine the rest of the core emission. The curvature of its trajectory will be magnified by projection, and its apparent velocity will exceed the speed of light. Of course the core will still appear brightest when the observer's line of sight is aligned with the precession cone axis. A source like 3C 273 could then show superluminal motion and a curved VLBI jet over about half of all possible orientations of the observer with respect to the source.

A potential observational problem for this secondary collimation model is that an observer whose line of sight lies inside the precession cone may see Doppler-boosted emission on the side of the core opposite of the large-scale jet.

\section{SUMMARY AND DISCUSSION}

Fueling of central massive black holes in galactic nuclei via tidal disruption of stars near the hole is a natural consequence of galactic cannibalism. It may account for the local space density of active galaxies and suggests that the steep cosmological evolution of AGNs is due to the rapid evolution of the merging rate of large galaxies during the collapse of rich clusters (Papers II and III, respectively). Binary black holes with separations of the order of one-tenth of the cusp radius will be formed as a result of mergers of two galaxies both containing a massive black hole. The rotation axes of both holes will perform geodetic precession about the total angular momentum of the binary. This may become manifest during periods of central activity in the form of precession of a radio (or optical) jet emitted along the rotation axis of the more massive hole (BBR). Such a period of activity may be triggered by subsequent mergers which will also cause a pre-existing central binary to lose orbital energy, due to interactions with stars passing through its orbit. As a result, the precession period of the central hole will decrease as the fueling rate increases. The fueling rate will remain relatively low for $\sim 10^{8}-10^{6}$ yr while the precession period decreases in the range $10^{9}-10^{6} \mathrm{yr}$. During the final most luminous stage of an $\mathrm{AGN}$, which lasts only $\sim 10^{4}-10^{6} \mathrm{yr}$, the precession period drops sharply and may become as short as a few hundred years. Such an object would exhibit strong curvature on the milli-arcsec scale and slow variation of position angle in the older arcsec scale structure (3C 273 and 3C 345 may be examples). The preexisting binary black hole will finally merge due to loss of orbital energy via the emission of gravitational waves, and a new stable binary is formed with a separation of again about one-tenth of the cusp radius. The same sequence of events is expected to take place in the nucleus of the intruding galaxy during the merging process, but the level of its maximal central activity will in most cases probably be considerably lower because its central black hole will be less massive (see Paper II).

The model thus predicts a correlation between precession period and level of central activity. A preliminary examination of available observational data indicates that radio galaxies have long precession periods, while periods shorter than $10^{5}$ yr occur only in AGNs that are more luminous at both radio and optical wavelengths.

In $\S \mathrm{V}$ we have mentioned several problems associated with rapid precession of relativistic jets in luminous quasars. As a possible solution to these problems we have mentioned collimation of the precessing jet material along the precession cone axis. We would then observe curvature due to precession and collimation on small (pc) scales, an almost straight (perhaps wiggling) jet on intermediate $(\mathrm{kpc})$ scales, and curvature due to slow precession on the largest scale.

In unified schemes for quasars, based on the relativistic beaming hypothesis (Scheuer and Readhead 1979; Orr and Browne 1982), the ratio $R$ of the core flux density over the flux density of the extended steep spectrum radio emission is determined by the orientation of the observer's line of sight with respect to the collimated relativistic material emitted from the core toward the outer radio lobes. At least two serious objections have been raised against the simple version of this model: (1) the difference in orientation between the large- and small-scale structures can be quite large (Schilizzi and de Bruyn 1983), (2) there is evidence that superluminal motion and Doppler boosting can be observed in a larger fraction of sources than the $\sim 1 / \gamma^{2}$ in simple beaming models (e.g., Kellerman 1986). Beamed jet emission over a larger range of angles may be due to velocity differences and shocks in the jet (Lind and Blandford 1985) or to a large opening angle of the jet itself (Rees 1981). Rapidly precessing jets in luminous quasars exhibit a larger range in jet directions and may also help to overcome the difficulties mentioned above. The statistical properties of rapidly precessing bright quasars would then be distinct from those of slowly precessing radio galaxies. For instance: (1) high values of $R$ (core flux density/flux density of extended component) will be more common among quasars (see, e.g., Ruiter, Rogara, and Padrielli, 1985), and (2) superluminal expansion velocities may be observed in up to $50 \%$ of all bright quasars, compared to a fraction $\gamma^{-2}$ of weaker radio galaxies.

At large redshifts we expect an increasing fraction of rapid precessors among quasars, since their mean luminosity increases. The distorted appearance of many steep spectrum quasars at large $z$ (Wilkinson et al. 1983; Barthel 1984) could be due to precession. The ambient medium could also play an important role, especially when most of these quasars reside in rich clusters of galaxies as predicted by models that connect the collapse of rich clusters with the cosmological evolution of quasars (Stocke and Perrenod 1981; De Robertis 1985; Papers I and III). There is now some observational evidence for a close association of quasars with regions of high galaxy density (e.g., Yee and Green 1987; Shaver 1987). A conclusion of this paper is that the central engine in bright quasars may be rapidly precessing. As a consequence we expect distorted radio structures, Doppler-boosted radio cores, and superluminal velocities to be common phenomena in high-redshift quasars.

I thank A. G. de Bruyn and E. J. A. Meurs for discussions and useful comments on the manuscript. The work was supported by a grant from the Leids Kerkhoven-Bosscha Fonds. 


\section{REFERENCES}

Bahcall, J. H., and Wolf, R. A. 1976, Ap. J., 209, 214.

Barthel, P. D. 1984, Ph.D. thesis, University of Leiden

Begelman, M. C., Blandford, R. D., and Rees, 'M. J. 1980, Nature, 287, 307 (BBR)

Biretta, J., Cohen M and Moore, R. 1986, IAU Symposium, 119, Quasars, ed. G. Swarup and V. K. Kapahi (Dordrecht: Riedel), p. 157

Blandford, R. D., and Königl, A. 1979, Ap. J., 232, 34.

Bridle, A. H., and Perley, R. A. 1984, Ann. Rev. Astr. Ap., 22, 319

Browne, I. W. A., Clark, R. R., Moore, P. K., Muxlow, T. W. B., Wilkinson, P. N. Cohen, M. H., and Porcas, R. W. 1982, Nature, 299, 788.

Bowne, I. W. A., and Perley, R. A. 1986, M.N.R.A.S., 222, 149.

Cohen, M. H., and Unwin, S. C. 1984, IAU Symposium 110, VLBI and Compact Radio Sources, ed. R. Fanti, K. Kellerman, and G. Setti (Dordrecht: Reidel), p. 95.

Condon, J. J., and Mitchell, K. J. 1984, Ap. J., 276, 472.

Davis, R. J., Muxlow, T. W. B., and Conway, R. G. 1985, Nature, 318, 343.

De Robertis, M. 1985, A.J., 90, 998.

de Ruiter, H. R., Rogora, A., and Padrielli, L. IAU Symposium 119, Quasars, ed.

G. Swarup and V. K. Kapahi (Dordrecht: Reidel), p. 197.

Gower, A. C., Gregory, P. C., Hutchings, J. B., and Unruh, W. G. 1982, Ap. J., 262, 478 .

Hills, J. G. 1975, Nature, 254, 295.

. 1983, A.J., 88, 1269 .

Hunstead, R. W., Murdock, H. S., Condon, J. J., and Philips, M. M. 1983, M.N.R.A.S., 207, 55 .

Icke, V. 1981, Ap. J.,(Letters), 246, L65.

Jones, D. L., et al. 1986, Ap. J., 305, 684.

Kellerman, K. I. 1986, IAU Symposium 121, Observational Evidence of Activity in Galaxies, ed. E. Ye. Khachikian, K. J. Fricke, and J. Melnick (Dordrecht Reidel), p. 273.

Lind, K. R., and Blandford, R. D. 1985, Ap. J., 295, 358.

Linfield, R. 1981, Ap. J., 250, 464.

Longair, M. S., and Ryle, J. M. 1979, M.N.R.A.S., 188, 625.

Miley, G. K. 1980, Ann. Rev. Astr. Ap., 18, 165.
Muxlow, T. W. B., Jullian, M., and Linfield, R., 1983, IAU Symposium 110, VLBI and Compact Radio Sources, ed. R. Fanti, K. Kellerman, and G. Setti (Dordrecht: Reidel, 1984), p. 141.

Oort, J. H. 1977, Ann. Rev. Astr. Ap., 15, 295.

Orr, M. J. L.. and Browne, I. W. A. 1982, M.N.R.A.S.. 200. 1067

Quinn, P. J., and Goodman, J. 1986, Ap. J., 309, 472.

Readhead, A. C. S., Cohen, M. H., Pearson, T. J., and Wilkinson, P. N. 1978, Nature, 276, 768.

Readhead, A. C. S., Hough, D. H., Ewing, M. S., Walker, R. C., and Romney, J. D. 1983, Ap. J., 265, 107.

Rees, M. J. 1981, in IAU Symposium, Origin of Cosmic Rays, ed. G. Setti, G. Spada, and A. W. Wolfendale (Dordrecht: Reidel), p. 139.

Rees, M. J. 1988, Nature, 333, 523

Roos, N. 1981a, Astr. Ap., 95, 349

. 1981b, Astr. Ap., 104, 218 (Paper I)

. 1985a, Ap. J., 294, 479 (Paper II)

1985b, Ap. J., 294, 486 (Paper III).

Roos, N., and Meurs, E. J. A. 1987, Astr. Ap., 181, 14

Scheuer, P. A. G., and Readhead, A. C. S. 1979, Nature, 277, 182.

Schilizzi, R. T., and de Bruyn, A. G. 1983, Nature, 303, 26.

Shaver, P. A. 1987, in IAU Symposium 130, Evolution of Large-Scale Structures in the Universe (Dordrecht: Reidel), in press.

Stocke, J. T., and Perrenod, S. C. 1981, Ap.J., 245, 375.

Toomre, A., and Toomre, J. 1972, Ap. J., 178, 623.

van der Laan, H., Zieba, S., and Noordam, J. E. 1984, in IAU Symposium 110 VLBI and Compact Radio Sources, ed. R. Fanti, K. Kellerman, and G. Setti (Dordrecht: Reidel), p. 9.

Veron-Cetty, M. P., and Veron, P. 1985, A Catalogue of Quasars and Active Nuclei (Geneva: ESO Sci. Rep.), p. 1.

Weinberg, S. 1972, Gravitation and Cosmology (New York: Wiley)

Wilkinson, P. N., Spencer, R. E. Readhead., A. C. S. Pearson, T. J., and Simon, R. S. 1984, in IAU Symposium 110, VLBI and Compact Radio Sources, ed. R. Fanti, K. Kellerman, and G. Setti (Dordrecht: Reidel), p. 25.

Yee, H. K. C., and Green, R. F. 1987, Ap. J., 319, 28.

Nico Roos: Sterrenkundig Instituut, Katholieke Universiteit Nijmegen, Toernooiveld, 6525 ED Nijmegen, The Netherlands 